\title{
LOS DETERMINANTES DE LA CONCENTRACIÓN ÉTNICA EN EL MERCADO LABORAL ESPAÑOL
}

\section{DETERMINANTS OF ETHNIC CONCENTRATION IN THE SPANISH LABOUR MARKET}

\author{
Alberto Veira aveira@polsoc.uc3m.es \\ Universidad Carlos III de Madrid. España.
}

Mikolaj Stanek mstanek@geps.es

Consejo Superior de Investigaciones Científicas. España.

\author{
LORENZO CACHóN Icachon@cps.ucm.es \\ Universidad Complutense de Madrid. España.
}

\begin{abstract}
RESUMEN
El principal propósito de este artículo es determinar qué mecanismos influyen en la concentración de la población inmigrante en determinadas ocupaciones del mercado de trabajo español. Mediante la exploración de los datos de la Encuesta Nacional de Inmigrantes (2007) identificamos los nichos en los que se agrupan los trabajadores procedentes de cinco países: Argentina, Ecuador, Francia, Marruecos y Rumania. En segundo lugar, mediante regresiones logísticas multinomiales contrastamos el impacto del capital humano, el capital social y variables contextuales sobre la probabilidad de que los inmigrantes se concentren en los principales nichos étnicos: los servicios de hostelería, el trabajo no cualificado en la agricultura, la construcción y el servicio doméstico. Los resultados del análisis indican que el nivel de estudios, el estatus legal, los contactos en el momento de llegada a España y la experiencia laboral anterior son importantes predictores de la presencia de los inmigrantes en los nichos ocupacionales mencionados.
\end{abstract}

\section{Palabras Clave}

España, Mercado laboral, Migraciones, Nicho étnico.

\section{RESUMEN}

This article provides insight on the mechanisms which determine the concentration of immigrant population in certain occupational categories in the Spanish labour market. Using survey data from the Spanish National Immigrant Survey (2007) we have firstly identified ethnic niches where Argentinean, Ecuadorian, French, Moroccan and Romanian workers concentrate. Secondly, we run multinomial logit models in order to assess the impact of human capital, social capital and contextual related variables on the likelihood of working in some of occupational categories identified previously as ethnic niches, namely: hospitality industry workers, routine agriculture workers, construction workers and domestic labour. Our main findings suggest that educational attainment, legal status, access to contacts at the time of arrival in Spain, country of origin, and previous job experience are strong predictors of the presence of foreign labour in the above mentioned niches.

\section{KEY WORDS}

Ethnic niche, Labour market, Migration, Spain. 


\section{INTRODUCCIÓN ${ }^{1}$}

Es bien sabido que los trabajadores inmigrantes se distribuyen de manera desigual en el mercado laboral (Castles y Miller 2003; Model 1997; Morawska 1990). La participación de los inmigrantes en determinados empleos adquiere en algunos casos un carácter marcadamente asimétrico, lo que permite confirmar la existencia de nichos étnicos ${ }^{2}$. Model (1993; véase también Waldinger 1996) propone considerar como nicho étnico aquella rama de actividad o categoría ocupacional en la que un colectivo supera al menos en un $50 \%$ su participación media en la fuerza de trabajo en el mercado laboral.

Aunque la existencia de nichos étnicos es un rasgo característico de la mayoría de países receptores de flujos migratorios, se observan importantes diferencias entre colectivos en cuanto a la escala de concentración y los sectores del mercado laboral que ocupan (Rath 2002). Los patrones de concentración étnica varían también en relación al contexto geográfico y a las condiciones económicas, sociales e institucionales específicas de cada país o región (Hudson 2003). Dado su peso en el conjunto de la fuerza de trabajo y su impacto sobre las políticas sociales, se ha prestado una mayor atención a los fenómenos de confinamiento de los inmigrantes en los puestos de trabajo caracterizados por unos ingresos reducidos, bajo prestigio y escasa deseabilidad social. No obstante, existe una abundante evidencia empírica que señala que la concentración étnica se produce también en algunos empleos que sitúan a determinados colectivos de inmigrantes en las partes superiores de la jerarquía ocupacional (Waldinger 1996; Wilson 2003) ${ }^{3}$. Por otra parte, se observa que varones y mujeres presentan distintos niveles de concentración y se agrupan en distintas ramas

\footnotetext{
${ }^{1}$ Este artículo se enmarca en el proyecto de investigación "Encuesta Nacional de Inmigrantes (ENI): Explotación con Perspectivas Comparativas Nacionales e Internacionales" financiado por el Ministerio de Ciencia e Innovación (CSO 2008-03616/SOCI). Los autores agradecen a Paul Schmelzer y Esther Ochoa Fernández sus valiosas sugerencias y consejos referentes al análisis estadístico realizado en este estudio.

${ }^{2}$ Es necesario recalcar que en los últimos años el análisis de la participación de inmigrantes y minorías étnicas en el mercado laboral ha aportado varios conceptos de entre los cuales destacan la economía étnica, enclave étnico y nicho étnico (Arjona y Checa 2006). La economía étnica describe un amplio fenómeno que abarca la actividad económica de los inmigrantes y miembros de minorías étnicas sea en forma de empresariado, gerencia de negocios o trabajo asalariado (Light 2007). El enclave étnico es un concepto más específico y designa la actividad económica de colectivos minoritarios que se caracteriza por un alto grado de concentración espacial, y el desarrollo de diversas formas de empresariado (Valdez 2006). A diferencia de los dos conceptos anteriormente mencionados, el nicho étnico se centra en el aspecto cuantitativo y se refiere al fenómeno de sobrerrepresentación numérica de los miembros de un grupo étnico en un sector de actividad o categoría ocupacional en relación al conjunto población empleada en estos trabajos.

${ }^{3}$ El ejemplo mas destacado es la alta concentración de los inmigrantes procedentes de algunos países asiáticos en los servicios TIC en la economía norteamericana (Hudson 2003; Wang 2004).
} 
y ocupaciones dentro del mercado de trabajo (Ellis y Wright 1999; Hudson 2003; Wright y Ellis 2000). En este sentido, como constatan algunos autores, los fenómenos de concentración étnica reproducen y refuerzan las desigualdades en la distribución ocupacional y sectorial entre la población masculina y femenina, generando así una doble segregación: étnica y por género (Bradley y Healy 2008; Schrover, Van der Leun y Quispel 2007).

También en España se observa el fenómeno de concentración étnica en determinadas ramas de la actividad económica (y ocupaciones dentro de ellas) como la construcción, la agricultura, el servicio doméstico y la hostelería (Cachón 2009; Cebolla y González 2008; Pajares 2010). Además de estar desigualmente distribuidos en el mercado de trabajo, los inmigrantes ocupan habitualmente posiciones en las partes inferiores de la escala sociolaboral, tienen menores probabilidades de movilidad ascendente y peores condiciones de trabajo en términos tanto de estabilidad laboral como de ingresos (Canal-Domínguez y Rodríguez-Gutiérrez 2008; Fernández y Ortega 2008; Martín Urriza 2006). Otra característica de la participación de los inmigrantes en el mercado laboral español es la existencia de profundas diferencias entre la población masculina y femenina en los patrones de inserción laboral (Del Río y Alonso-Villar 2010; Parella 2003; Vidal, Gil y Domingo 2009).

En los últimos años el número de estudios dedicados a la problemática de los procesos de concentración y segmentación de la fuerza laboral extranjera en el mercado de trabajo español ha incrementado considerablemente (véase por ejemplo: Alcobendas y Rodríguez-Planas 2009; Bernardi, Garrido y Miyar 2011; Cachón 2009; Cuadrado, Iglesias y Llorente 2007; Domingo, Gil-Alonso y Robertson 2007; Simón, Sanromá y Ramos 2008). No obstante, consideramos necesario profundizar nuestro conocimiento sobre los factores que determinan la inserción de la población inmigrante en los nichos étnicos característicos del mercado laboral español. Además, consideramos que la complejidad del fenómeno de la concentración étnica en el mercado laboral obliga a los investigadores no sólo a plantear preguntas sobre los determinantes generales de la incorporación de inmigrantes en los nichos ocupacionales, sino también a indagar sobre las causas de la diversidad de patrones que adquiere este fenómeno en relación al género, el tipo de empleo y el país de origen. Por lo tanto, el primer objetivo de este artículo es el de establecer las principales pautas de concentración de cinco colectivos inmigrantes originarios de Francia, Rumania, Marruecos, Ecuador y Argentina, e identificar cuáles son los nichos que ocupan en el mercado laboral español a partir de los datos de la Encuesta Nacional de Inmigrantes 2007. Posteriormente, mediante un análisis logístico multinomial, se identifican los predictores de la participación de la población inmigrante en los principales nichos étnicos (hostelería, construcción, servicio doméstico y agricultura). En nuestro estudio adoptamos una doble perspectiva. En primer lugar, estamos interesados en determinar si los factores que determinan la inserción en los nichos seleccionados operan de una forma similar. En segundo lugar, realizando análisis por separado para la población masculina y femenina, pretendemos identificar factores específicos de concentración étnica en relación al género. 


\section{APROXIMACIONES TEÓRICAS E HIPÓTESIS}

\section{Principales enfoques de análisis de los nichos étnicos}

Dada la variedad de modos y contextos en los se produce el fenómeno de la concentración étnica no es de extrañar que se hayan planteado diversos marcos explicativos, de los cuales, los más destacadas son la teoría del capital humano, el enfoque del capital social y la teoría de segmentación de mercados.

La teoría del capital humano interpreta el fenómeno del desarrollo de los nichos étnicos dentro del mercado laboral como resultado de un ajuste entre la oferta de mano de obra de determinadas características y la demanda de empleo por parte de las empresas. Los distintos modos en los que los inmigrantes se insertan en el mercado laboral receptor responden a las diferencias en la productividad y a desigualdades en las dotaciones de capital humano. Bajos niveles de preparación, desconocimiento del idioma de país de acogida o una limitada experiencia laboral constituyen factores que sitúan a algunos inmigrantes en desventaja, relegándolos a las posiciones inferiores de la escala ocupacional (Chiswick, Lee y Miller 2003). Otro de los factores que determinan la situación de los inmigrantes en el mercado laboral es el grado de transferibilidad de las cualificaciones obtenidas en el extranjero. Las diferencias en los modos de incorporación laboral entre inmigrantes de distintas procedencias que, al menos formalmente, poseen el mismo nivel de preparación se deben también a las diferencias en la compatibilidad de sistemas educativos entre los países de origen y destino (Friedberg 2000). Además, como señalan Raijman y Semyonov (1995), algunas ocupaciones (por ejemplo las referentes a técnicos e ingenieros) se ajustan mejor a la demanda en el mercado laboral receptor.

En las últimas décadas se observa el florecimiento de estudios dedicados al impacto que tiene el capital social en la situación laboral de los inmigrantes. El capital social se puede definir como la capacidad de los actores para asegurarse beneficios en virtud de su pertenencia a las redes sociales o a otras estructuras sociales (Portes 1998). En otras palabras, el capital social es un atributo del individuo que consiste en el acceso a varias clases de recursos (información, ayuda económica, apoyo psicológico) a través de distintos tipos de relaciones sociales. Los recursos a los que tienen acceso los inmigrantes se generan tanto en el marco de las redes sociales personales basadas en lazos familiares y de amistad como en las estructuras de las relaciones sociales menos inmediatas, tales como la participación en diversas entidades u organizaciones de todo tipo (asociaciones étnicas, grupos religiosos, grupos deportivos etc.) (Massey et al. 1987).

El capital social es considerado un recurso importante para la inserción laboral en la economía del país receptor, sobre todo, cuando los inmigrantes desconocen el idioma, disponen de un limitado capital humano o sus características no permiten su uso en el contexto del mercado laboral en el país de destino (Green, Tigges y Diaz 1999; Sanders, Nee y Sernau 2002). Desde este punto de vista, la dependencia de los inmigrantes de las redes sociales es un factor crucial en los procesos de concentración étnica en 
determinados sectores del mercado laboral, ya que constituyen una importante fuente de información acerca de las oportunidades de empleo. En consecuencia, las relaciones sociales basadas en un menor número de contactos y de menor heterogeneidad aumentarían la probabilidad de inserción en los nichos laborales (Ooka y Wellman 2003; Pascual, De Miguel y Solana 2007: 21).

Según el enfoque de la segmentación laboral, el mercado laboral esta dividido en, al menos, dos segmentos muy diferentes en cuanto a las reglas de organización de trabajo, los mecanismos de asignación laboral y movilidad ocupacional y las condiciones laborales. El mercado primario ofrece empleos estables, salarios relativamente altos, aceptables condiciones de trabajo y posibilidades de promoción ocupacional. El sector secundario, en cambio, se caracteriza por un uso intensivo de mano de obra con bajos salarios, inestabilidad contractual y limitadas oportunidades para la promoción ocupacional (Piore 1979). La baja aceptabilidad de los empleos en el sector secundario por parte de los trabajadores nativos, las barreras estructurales que impiden la mejora de las condiciones de contratación y la creciente incorporación de la población femenina autóctona en los segmentos primarios generan una continua demanda de mano de obra extranjera en actividades del sector secundario. Los inmigrantes se ven relegados a los puestos de trabajo en este sector, independientemente de su nivel de preparación y de su experiencia laboral previa, lo que tiene como consecuencia la concentración étnica en determinadas ocupaciones tales como el servicio doméstico, la construcción y la agricultura (Pedace 2006).

Además, la perspectiva del enfoque de la segmentación mantiene que la segregación étnica en los mercados laborales se intensifica en los periodos de un mayor crecimiento económico, cuando los trabajadores nativos encuentran mayores posibilidades de incorporarse en los puestos de trabajo de mayor prestigio y mejor renumerados, dejando vacantes los empleos en el sector secundario (McGovern 2007). Algunas de las aportaciones en el marco de este enfoque enfatizan el papel del Estado en los procesos de segmentación de mano de obra extranjera. Según diversos autores, las normas y prácticas administrativas crean un "marco institucional discriminatorio" (por ejemplo,. mediante las restricciones en el acceso a los permisos de residencia y trabajo, limitaciones de los mismos a determinadas actividades al menos de modo inicial o dificultades en las homologaciones de los títulos académicos) que limitan el acceso de los inmigrantes a los empleos fuera del sector secundario, respondiendo así a las demandas específicas de un sector del empresariado (Abad 2002; Cachón 2009).

\section{Hipótesis del estudio}

Partiendo de estas tres perspectivas se han planteado las siguientes hipótesis de trabajo:

(1) Según las permisas del enfoque del capital humano podemos esperar que los inmigrantes con pocos estudios, deficiente conocimiento del idioma español y cuyas cualificaciones se caracterizan por una baja transferibilidad estén más expuestos al riesgo concentrarse en los nichos laborales de menor prestigio y bajos ingresos. 
(2) Desde la perspectiva del enfoque del capital social es de esperar que los inmigrantes que declaran haber contado con el apoyo de amigos o familiares al llegar a España se inserten con mayor probabilidad en alguno de los nichos laborales. Por otra parte, las personas que tienen acceso a recursos sociales más amplios y diversos (es decir, mediante participación en redes sociales de mayor heterogeneidad étnica y social) pueden estar expuestas en menor medida a los procesos de concentración étnica.

(3) Finalmente, la perspectiva de segmentación sugiere que residir en situación irregular en España y llegar en el periodo de mayor crecimiento económico aumenta el riesgo de encontrar empleo en un nicho étnico.

\section{FuENTES DE DATOS Y METODOLOGíA}

\section{Fuentes de datos y marco muestral}

Los análisis que se presentan en este estudio se basan en la Encuesta Nacional de Inmigrantes (ENI-2007) y, en menor medida, en la Encuesta de Población Activa (primer trimestre de 2007) 4 . Aunque la ENI-2007 cuenta con 15.465 personas encuestadas, la muestra utilizada en nuestro estudio ha sido restringida a las personas ocupadas en el momento de la realización de la encuesta, con edades comprendidas entre 20 y 65 años y llegados a España a partir de $1989^{5}$, es decir, ha sido acotada a 6.552 casos.

\section{Método y variables}

El principal instrumento analítico utilizado para contrastar las hipótesis planteadas ha sido la regresión logística multinomial. La variable dependiente ha sido construida a partir de la clasificación de ocupaciones incluida en la base de datos ENI-2007. Como modo de establecer el grado de concentración en cada una de 19 categorías ocupacionales se ha aplicado la razón de probabilidades (odds ratio) ${ }^{6}$. La decisión de aplicar esta medida fue motivada por su especial utilidad en el análisis comparativo entre grupos de

\footnotetext{
${ }^{4}$ Los datos de la ENI-2007 y de la EPA-2007 han sido utilizadas conjuntamente para el cálculo de índices de concentración laboral e identificación de los nichos étnicos. En cambio los modelos multinomiales en este estudio están basados exclusivamente en los datos de la ENI-2007.

${ }^{5}$ Esta restricción se debe a que una de las variables referentes al capital social (en concreto la correspondiente a la pregunta: ¿Tenía a alguien a quien dirigirse cuando llegó a España?) fue dirigida exclusivamente a las personas que llegaron a España después de 1989.

${ }^{6}$ En término generales, la razón de probabilidades designa el coeficiente entre la probabilidad de que ocurra un suceso $(p)$ y la probabilidad de que no ocurra $(1-p)$.
} 
diferentes características y tamaños (Wang y Pandit 2007). Su eficacia fue confirmada en numerosas investigaciones realizadas sobre todo en el contexto norteamericano (Rosenfeld y Tienda 1999; Wilson 1999).

Para el caso de la concentración étnica, la razón de probabilidades se obtiene calculando el coeficiente entre la probabilidad de que un miembro del colectivo inmigrante se encuentre en una determinada categoría ocupacional y la misma probabilidad para las personas ocupadas no pertenecientes a este colectivo:

$$
R P=\left(M_{t} / M_{1-t}\right) /\left(O_{t} / O_{1-t}\right)
$$

En la formula, el numerador es la razón entre el número de inmigrantes de un colectivo en determinada ocupación $\left(\mathrm{M}_{\mathrm{t}}\right)$ y el número de inmigrantes del mismo origen empleados en otras categorías ocupacionales $\left(\mathrm{M}_{1-1}\right)$. El denominador representa la razón entre los trabajadores pertenecientes a otros colectivos (nativos y de otros grupos inmigrantes) que se encuentran en una determinada categoría ocupacional $\left(O_{t}\right)$ y los trabajadores de estos orígenes que trabajan en otras ocupaciones $\left(\mathrm{O}_{1-1}\right)$ (Wang y Pandit 2007; Wilson 2003).

El valor 1 indica que el grado de participación ocupacional de un colectivo étnico se asemeja al de resto de la población en su conjunto. En este contexto, es necesario establecer el valor a partir del cual se considere que la sobrerrepresentación de un grupo inmigrante en determinado empleo adquiere rasgos de nicho laboral. Siguiendo la ya citada definición de Model se ha fijado en 1,5 el nivel mínimo de la razón de probabilidades$^{7}$. Finalmente, con el propósito de evitar los sesgos debidos al reducido número de casos presentes en algunas categorías se ha considerado como nicho étnico aquellos empleos en los que el número trabajadores no es inferior al $50 \%$ del número medio de inmigrantes de este origen distribuidos en las 19 categorías ocupacionales analizadas (véase Wang y Pandit 2007; Wilson 1999).

Partiendo de la premisa sobre las diferencias en cuanto a los factores de concentración laboral basados en el género, la estimación de los predictores de inserción en los nichos étnicos se ha realizado por separado para varones y mujeres. Contamos con cuatro categorías en cada modelo: peones de la agricultura, trabajadores de los servicios de hostelería y servicio doméstico en el modelo de las mujeres, y peones de la agricultura, trabajadores de los servicios de hostelería y trabajadores de la construcción en el modelo diseñado para los varones. En ambos casos la categoría de referencia son el "resto de ocupaciones". A partir de cada uno de los modelos estimamos la probabilidad de que un trabajador pueda estar en uno de los nichos frente a la categoría de referencia.

En nuestros modelos hemos introducido una serie de variables sociales y demográficas tales como la procedencia del inmigrante y la incidencia de la cohabitación. Se ha

\footnotetext{
${ }^{7}$ Este valor es el más utilizado en los análisis de concentración étnica (Logan et al. 2000; Wang 2004; Wilson 2003), no obstante en algunos estudios el umbral a partir el cual se define el nicho étnico se fija en 1,2 02 (Ellis y Wright 1999; Hudson 2003)
} 
prestado especial atención al efecto de pertenecer a alguno de los cinco colectivos de extranjeros cuyo peso es mayoritario en el conjunto de la población inmigrante ocupada en España: rumanos, marroquíes ecuatorianos, argentinos y franceses ${ }^{8}$.

Como ya se ha dicho, el principal objetivo del análisis es estimar el efecto de los factores que recogen las hipótesis planteadas en relación a los tres marcos explicativos. Los aspectos fundamentales del capital humano están recogidos por el nivel de estudios y dominio declarado del castellano. El grado de transferibilidad de cualificaciones se mide mediante la variable que refleja el hecho de haber convalidado la titulación académica obtenida en el país de origen.

Como medida del impacto de las relaciones sociales en la inserción en los nichos étnicos se ha utilizado la variable que recoge los contactos que tenía el inmigrante al llegar a España. Otro indicador del capital social incluido en los modelos es la participación en entidades 0 asociaciones dirigidas al público general. Suponemos que las personas que participan en este tipo de actividades disponen de unos recursos sociales más diversificados, lo que reduciría el riesgo de inserción en un nicho laboral.

Los predictores de la participación en un nicho étnico derivados de la perspectiva de la segmentación son la situación legal y el periodo de llegada. En primer lugar, suponemos que la situación irregular es proxy del "marco institucional discriminatorio". En el segundo caso, suponemos que los inmigrantes que llegaron en el periodo del boom económico en España (años 2002-2007) están más expuestos a encontrarse en un nicho étnico ${ }^{9}$. Asumimos aquí que en los periodos de mayor crecimiento económico los trabajadores nativos tienden a trasladarse a empleos en el sector primario, dejando puestos vacantes en los sectores secundarios que serían ocupados por los inmigrantes.

Los modelos también contienen dos variables de control que miden el efecto de la experiencia laboral previa. La primera identifica aquellos trabajadores que en su país de origen se encontraban trabajando en un empleo de características similares a los que en España constituyen un nicho. Suponemos que en estos casos los inmigrantes tienen una probabilidad considerablemente más elevada de encontrar empleo en la ocupación que ya habían realizado con anterioridad. La segunda recoge si el empleo actual es el primero que ha tenido el inmigrante tras su llegada a España.

\footnotetext{
${ }^{8}$ Los trabajadores rumanos suponen un $11,1 \%$ de la población inmigrante ocupada en España, los marroquíes un $10,2 \%$, los ecuatorianos un $9,7 \%$, los argentinos un $5,3 \%$ y los franceses un $4,5 \%$. Con el propósito de diversificar la muestra en cuanto a los marcos geográficos de procedencia se ha optado por excluir a los colombianos (quienes representan el 7,3\% de la población inmigrante ocupada) e incluir a los franceses.

${ }^{9}$ En este punto es necesario indicar que la época de mayor prosperidad económica es también la más reciente (en relación con la fecha de la ENI que es 2007) lo que puede sugerir que la entrada en los nichos está condicionada no tanto por la coyuntura económica sino por el hecho de que los últimos inmigrantes en llegar llegados sufren un mayor riesgo de encontrarse en determinadas ocupaciones. No obstante, este efecto está controlado por la variable que recoge la pregunta "¿Este es su primer trabajo en España?" (véase Stanek y Veira 2009).
} 


\section{Pautas de CONCENTRACIÓN ÉtNICA - ANÁLISIS DESCRIPTIVO}

\section{Distribución ocupacional}

La tabla 1 pone de relieve hasta qué punto la presencia en España de un elevado número de trabajadores de origen extranjero ha dado lugar a una nueva forma de segregación del mercado laboral en función del lugar de origen, que también reproduce y refuerza la segregación por razón de género. Si comparamos a la población española con la de origen extranjero comprobaremos que existen importantes diferencias que confirman las observaciones realizadas en otros estudios (Cachón 2009; Garrido y Toharia 2004). En el conjunto de las principales categorías de trabajadores de "cuello blanco" formado por los directores y gerentes de empresas, los profesionales universitarios y los técnicos de apoyo encontramos al $38,6 \%$ de varones y al $54,9 \%$ de mujeres españolas, frente a un $21,4 \%$ de varones y un $26,8 \%$ de mujeres de origen extranjero. Otras categorías ocupacionales en las que se aprecian importantes diferencias son la construcción (que da trabajo a un $32,4 \%$ de los varones extranjeros, frente a un $17 \%$ de varones españoles), el trabajo doméstico (en el que se emplean hasta un $23,2 \%$ de las mujeres de origen extranjero frente a un $3,3 \%$ de las españolas) y la hostelería (que emplea al 2,6\% y 4,5\% de varones y mujeres españoles frente a un $7,2 \%$ y $14 \%$ de varones y mujeres de origen extranjero).

La distribución ocupacional varía según el origen de los diferentes colectivos de inmigrantes. Las diferencias (y también las similitudes) entre cinco de los colectivos de inmigrantes más numerosos presentes en España se tornan evidentes observando las cifras de la tabla 1. La débil presencia de franceses y argentinos en el sector de la construcción contrasta con la fuerte implantación de ecuatorianos, marroquíes y, sobre todo, de rumanos. Entre las mujeres destaca el peso de las rumanas empleadas en el servicio doméstico, lo que también se observa, aunque en menor medida, entre las marroquíes, ecuatorianas y argentinas. En cuanto la hostelería, resulta evidente que esta ocupación atrae a un grupo relativamente elevado de mujeres rumanas, marroquíes, ecuatorianas y de varones y mujeres argentinos.

\section{Grado de segregación}

Una forma de resumir el nivel de segregación ocupacional que experimentan los trabajadores de origen extranjero en función de su lugar de procedencia es mediante índice de disimilitud. Esta medida refleja, para cada grupo poblacional el porcentaje de personas que deberían ocupar empleos diferentes a los que tienen para que la distribución fuese estadísticamente equivalente a la de la población autóctona ${ }^{10}$. La tabla 1 muestra los

\footnotetext{
${ }^{10}$ Aunque fue creado para medir la asimetría en la distribución espacial de una población minoritaria, en los últimos años ha encontrado utilidad en el análisis de movilidad y segregación socio-laboral (Martori y Hoberg 2004; Merschrod 1981).
} 


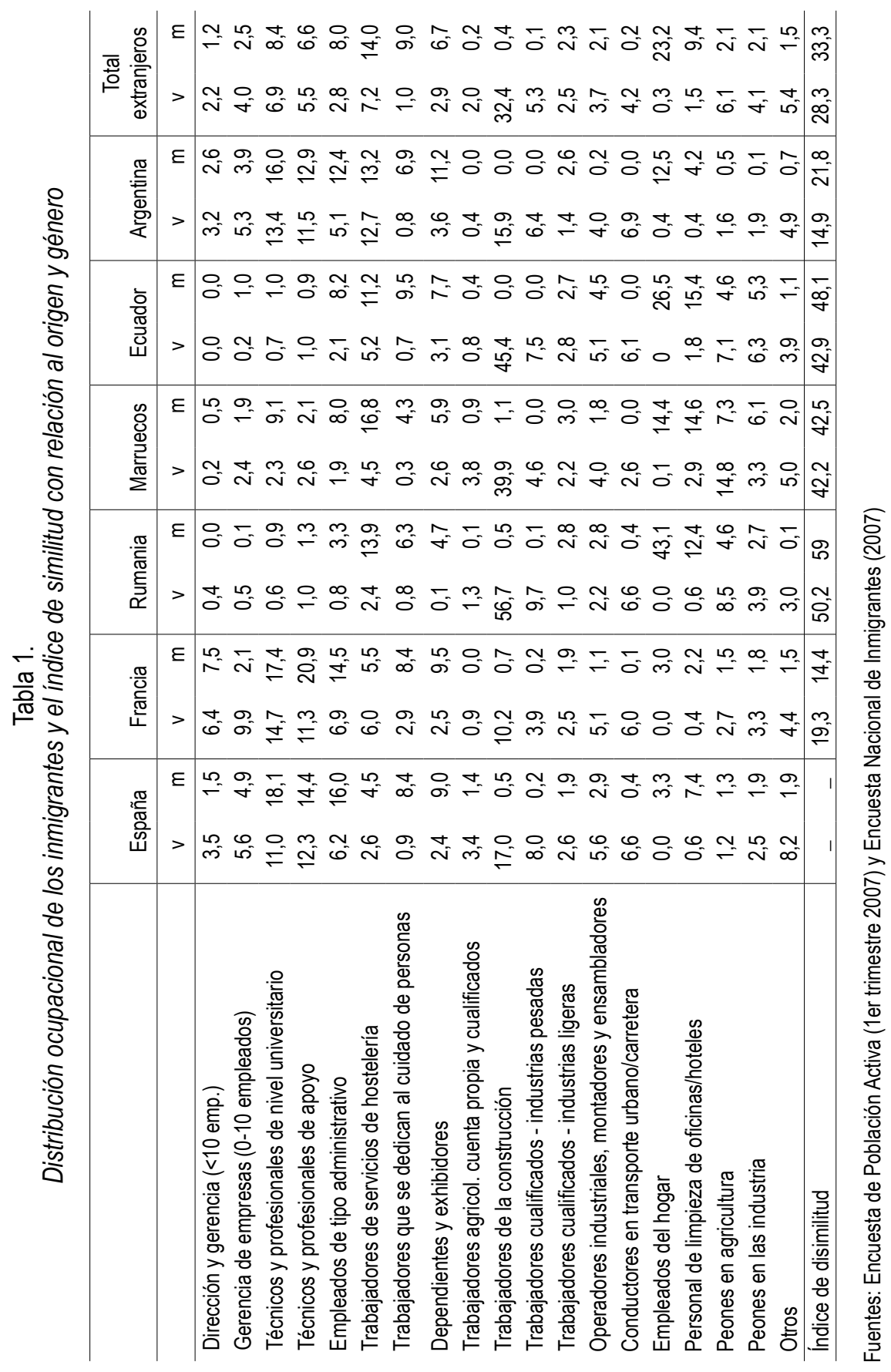


indices de disimilitud de las mujeres y varones de origen extranjero con respecto a los varones y mujeres nacidos en España. La necesidad de elaborar índices específicos para cada sexo viene dada por el hecho de que dentro de la propia población autóctona española se da una marcada segregación ocupacional por razón de género. Este indicador señala que hasta un $28,3 \%$ de los varones extranjeros deberían reubicarse en ocupaciones diferentes para que su distribución se asemejase a la de los varones autóctonos. Según este índice, las mujeres extranjeras sufren un nivel de segregación ocupacional todavía mayor que los varones, pues hasta un $33,3 \%$ deberían cambiar de ocupación para presentar una distribución ocupacional similar a la de las españolas ${ }^{11}$. Cuando se analizan de modo separado por nacionalidad puede verse que los mayores índices de disimilitud corresponden a los trabajadores rumanos, seguidos de los ecuatorianos y marroquíes.

\section{Nichos étnicos}

En la tabla 2 encontramos información sobre las probabilidades que tienen los inmigrantes de encontrarse en cada una de las ocupaciones en relación al resto de los trabajadores, permitiendo así determinar qué empleos son específicos para cada uno de los grupos poblacionales estudiados. Las casillas sombreadas indican las categorías ocupacionales que responden a los criterios de nicho étnico expuestos en la sección anterior. Si relacionamos la distribución ocupacional de los inmigrantes recogida en la tabla 1 con los datos sobre los nichos de la tabla 2 se ponen de manifiesto las diferencias entre la población femenina y masculina, siendo los varones inmigrantes los que en mayor proporción se insertan en nichos étnicos ( $50 \%$ frente a $37 \%$ en las mujeres).

$\mathrm{Si}$ atendemos a los datos referidos a las diferentes nacionalidades, apreciamos que en el caso de franceses y argentinos el peso de la población insertada en los nichos es considerablemente menor que en el resto de grupos. Los argentinos constituyen el colectivo con menor proporción de personas empleadas en los nichos (12,5\% los varones y $25,8 \%$ las mujeres). Por su parte, tan sólo uno de cada tres varones franceses está empleado en alguna de las categorías ocupacionales identificadas como nichos étnicos. En cuanto a los ecuatorianos y marroquies, hasta un $60 \%$ se concentran en nichos étnicos, con independencia del sexo. Finalmente, se observa que son los trabajadores procedentes de Rumania quienes experimentan el mayor grado de concentración étnica, pues hasta tres de cada cuatro rumanas y dos de cada tres rumanos trabajan en nichos étnicos.

${ }^{11}$ Como sugiere Massey (2002), los valores del índice por debajo de 30\% indican un grado de segregación bajo, los valores entre 30 y $59 \%$ una segregación moderada y por encima de este valor una segregación alta. 


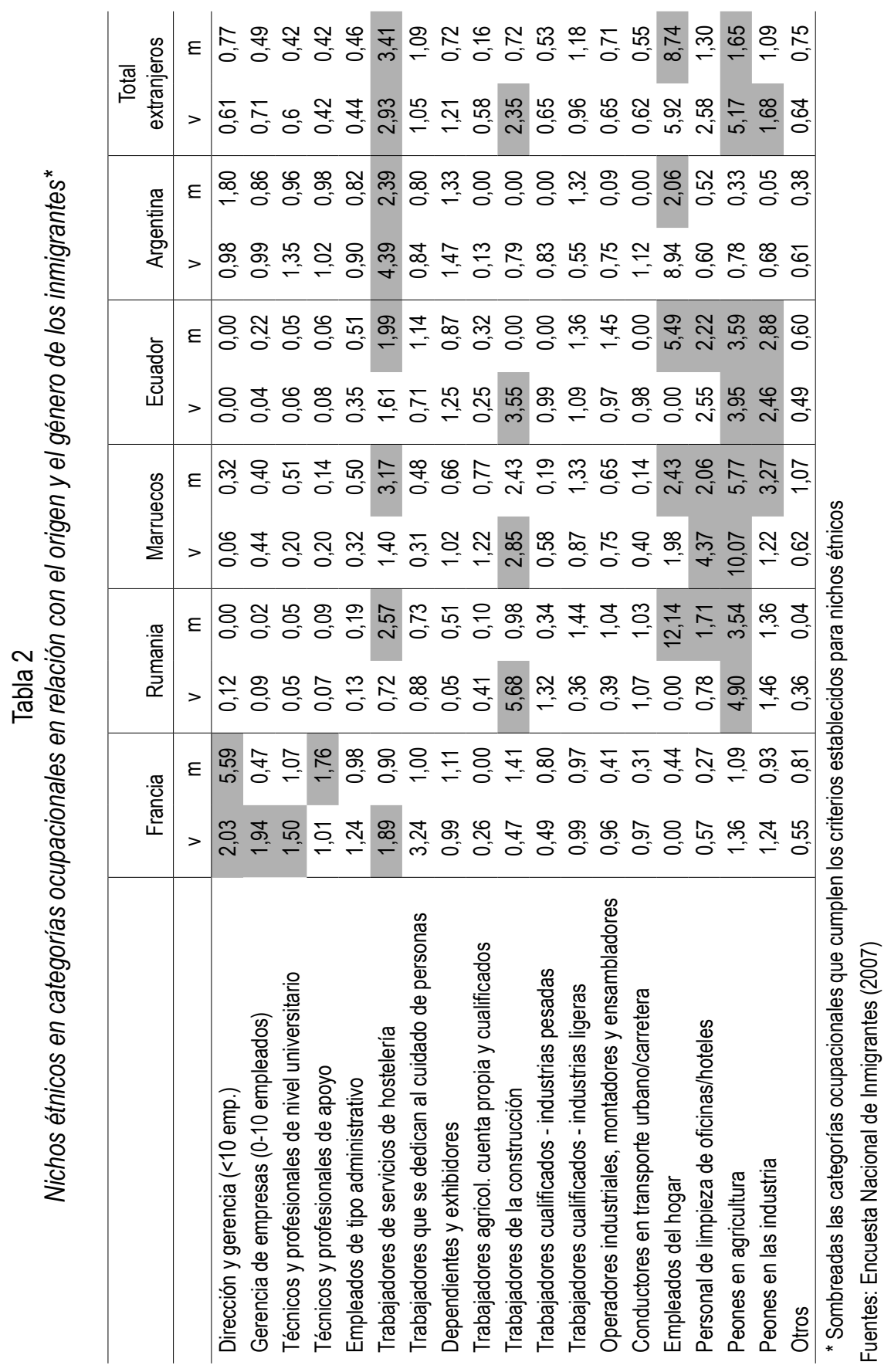


La tabla 2 confirma la existencia de claras diferencias en las pautas de concentración. Con relación al conjunto de la población ocupada en España, los inmigrantes franceses se concentran en empleos de "cuello blanco", con la única excepción de los servicios hosteleros, los cuales se han convertido en un nicho étnico para los varones de esta procedencia. En cuanto a la población de origen argentino, ésta tiende a concentrarse en los servicios hosteleros así como en empleo en el servicio doméstico. Observamos importantes similitudes entre los inmigrantes ecuatorianos, marroquíes y rumanos tanto en relación a los nichos que ocupan como a las pautas de segregación ocupacional por género. Los varones de estas procedencias han creado nichos de carácter étnico en la construcción. Por su parte, los nichos "típicamente" femeninos para estos tres colectivos son los servicios de hostelería, el servicio doméstico y la limpieza en oficinas/hoteles (nótese que los varones marroquíes también se especializan en este tipo de actividad). El único nicho común a varones y mujeres de estos tres países lo constituye el trabajo no cualificado en agricultura.

\section{RESULTADOS DEL ANÁLISIS MULTINOMIAL}

Los análisis multinomiales cuyos resultados se recogen en las tablas 3 y 4 presentan el efecto de las variables independientes sobre la probabilidad de trabajar en cada uno de esos nichos étnicos frente a la probabilidad de trabajar en alguna otra ocupación no identificada como nicho étnico. La tabla 3 resume los resultados para los varones en las ocupaciones relacionadas con los servicios de hostelería, el trabajo agrícola no cualificado y la construcción. La tabla 4 resume los resultados para las mujeres, e indica el papel que juegan los factores sobre las probabilidades de que éstas se empleen en los servicios de hostelería, como peones agrícolas o como empleadas del hogar.

Los resultados muestran que la hostelería atrae a los inmigrantes de cualquier nivel de estudios salvo los que poseen estudios universitarios. Entre las mujeres, carecer de una convalidación del título académico también parece ser un factor que favorece la entrada en este nicho. Sorprendentemente, el dominio del castellano no resulta ser una variable que determine la empleabilidad de los inmigrantes en esta ocupación. Disponer o no de contactos en España en el momento de llegar no es un factor que influya en el acceso a esta ocupación. Por el contrario, al menos entre las mujeres, la participación en entidades 0 asociaciones de atención al público resulta un factor asociado negativamente con encontrase empleado en este nicho laboral. Sobre el papel del marco laboral, cabe señalar que los indicios disponibles apuntan a que son los inmigrantes de llegada más reciente, es decir, los llegados durante el periodo de mayor crecimiento económico, los más propensos a encontrarse ocupados en la hostelería, especialmente entre la población masculina. La situación documental resulta irrelevante entre los hombres, pero entre las mujeres se aprecia que las que trabajan en la hostelería tienen una probabilidad mucho mayor de estar en posesión de un permiso de residencia que las empleadas en el resto de ocupaciones. Otros hallazgos de interés sobre este nicho 
laboral es que atrae en mayor medida a inmigrantes que no viven en pareja y que ya han tenido algún empleo anterior en España.

En el caso de los peones agrícolas las variables que miden el capital humano se comportan de forma esperada, o bien no arrojan resultados estadísticamente significativos. Tal y como cabría suponer, esta categoría ocupacional tiende a concentrar personas sin estudios 0 con estudios primarios, y, entre las mujeres, también a aquellas con estudios a nivel de secundaria de primer grado. También resulta claro que, entre los hombres, el carecer de una convalidación de los estudios obtenidos en el país de origen propicia la entrada en este nicho. En cuanto al dominio del castellano, no parece ser un factor que influya de manera decisiva sobre la probabilidad de emplearse o no en la agricultura. Sobre el papel del capital social, los resultados no son igual de concluyentes para las poblaciones masculina y femenina, pero en ambos casos los signos de los coeficientes indican que quienes se emplean como peones agrícolas carecen de contactos al llegar a España (sobre todo las mujeres) y tampoco desarrollan actividades en alguna entidad o asociación. Nuestros análisis indican, además, que haber llegado a España en la época de mayor prosperidad aumenta las posibilidades de que un inmigrante se encuentre empleado en la agricultura. También señalan que, en el caso de los varones, la agricultura resulta ser un nicho laboral que concentra una elevada proporción de personas sin permiso de residencia. Entre los hombres, el no vivir en pareja es un factor estrechamente relacionado con una mayor probabilidad de trabajar en este nicho.

La construcción es un nicho ocupacional casi exclusivo de la población masculina, por lo que el análisis multinomial se ha llevado a cabo utilizando únicamente los inmigrantes varones de la muestra. Los resultados muestran que las personas que se ocupan en este nicho no tienen una mayor o menor probabilidad de estar conviviendo con sus parejas que el resto de la población inmigrante. Los resultados también muestran que, al igual que sucede en el sector hostelero, quienes ya cuentan con una cierta experiencia laboral en España tienen mayores probabilidades de encontrar trabajo en la construcción. Hemos comprobado que este nicho ocupacional atrae fundamentalmente a inmigrantes sin estudios o con estudios primarios únicamente. También concentra a quienes no poseen una convalidación del título académico logrado en el país de origen. Por otra parte, el dominio del castellano no resulta un factor que aumente o disminuya las probabilidades de un inmigrante de emplearse en la construcción. Existen indicios de que el acceso a contactos al llegar España favorece la entrada de los varones en los empleos relacionados con la construcción. Por el contrario, trabajar en este nicho se encuentra negativamente asociado a la participación en asociaciones y entidades de atención al público. La construcción, al igual que la agricultura, resulta especialmente atractiva a aquellos inmigrantes de llegada reciente y con una situación documental irregular. No obstante los niveles de irregularidad no son tan altos como entre los peones agrícolas.

Los análisis sobre los factores que propician la entrada en el nicho laboral del trabajo doméstico se han restringido a la población femenina, por tratarse de una ocupación en la que la presencia de varones es mínima. De las cinco nacionalidades objeto de estu- 
Tabla 3.

Resultados de los análisis multinomiales para los inmigrantes varones

\begin{tabular}{|c|c|c|c|c|c|}
\hline & & & Hostelería & $\begin{array}{l}\text { Peones } \\
\text { agrícolas }\end{array}$ & Construcción \\
\hline Variables independientes & Casos & $\%$ & $\beta$ & $\beta$ & $\beta$ \\
\hline \multicolumn{6}{|l|}{ País de origen } \\
\hline Francia & 66 & 2,0 & 0,412 & 0,419 & $-1,779 * *$ \\
\hline Rumanía & 433 & 13,1 & $-1,312$ ** & 1,130 ** & $0,855^{* *}$ \\
\hline Marruecos & 397 & 12,0 & $-0,556+$ & $1,187^{\star *}$ & $0,282+$ \\
\hline Argentina & 203 & 6,1 & 0,152 & $-1,814+$ & $-0,658^{* *}$ \\
\hline Ecuador & 433 & 13,1 & $-0,601+$ & 1,289 ** & 0,654 ** \\
\hline Resto de inmigrantes (cat. ref.) & 1779 & 53,7 & & & \\
\hline \multicolumn{6}{|l|}{ Vive en pareja } \\
\hline Sí & 2126 & 64,2 & $-0,594$ ** & $-0,894$ ** & $-0,152$ \\
\hline No (cat. ref.) & 1185 & 35,8 & & & \\
\hline \multicolumn{6}{|l|}{ Ocupación en origen } \\
\hline Hostelería & 179 & 5,4 & 2,261 ** & $-0,895$ & 0,173 \\
\hline Agricultura & 191 & 5,8 & $-1,093$ & $1,630^{* *}$ & 0,426 * \\
\hline Construcción & 578 & 17,5 & $-0,402$ & $0,470^{*}$ & $1,465^{* *}$ \\
\hline Otra ocupación (cat. ref.) & 2363 & 71,4 & & & \\
\hline \multicolumn{6}{|l|}{ Es este su primer trabajo en España } \\
\hline Sí & 1084 & 32,7 & $-0,512^{* *}$ & 0,079 & $-0,284$ ** \\
\hline No (cat. ref.) & 2227 & 67,3 & & & \\
\hline \multicolumn{6}{|l|}{ Nivel de estudios } \\
\hline Sin estudios & 371 & 11,2 & 0,035 & $0,841^{* *}$ & 0,629 ** \\
\hline Primarios & 528 & 15,9 & 0,138 & 0,929 ** & 0,424 ** \\
\hline Secundarios $1 \mathrm{er}{ }^{0}$ & 506 & 15,3 & $-0,075$ & 0,327 & 0,158 \\
\hline Secundarios $2^{\circ}$ (cat. ref.) & 1304 & 39,4 & & & \\
\hline Universitarios & 602 & 18,2 & $-0,817$ ** & $-1,056^{* *}$ & $-0,678$ ** \\
\hline \multicolumn{6}{|l|}{ Estudios en España o titulo convalidado } \\
\hline Sí & 305 & 9,2 & $-0,069$ & $-0,416$ & $-0,775^{\star *}$ \\
\hline No (cat. ref.) & 3006 & 90,8 & & & \\
\hline \multicolumn{6}{|l|}{ Domina el español } \\
\hline Sí & 2721 & 82,2 & 0,200 & $-0,596$ ** & $-0,142$ \\
\hline No (cat. ref.) & 590 & 17,8 & & & \\
\hline \multicolumn{6}{|l|}{ Tenía contactos al llegar a España } \\
\hline Sí & 2583 & 78,0 & 0,256 & $-0,253$ & $0,177+$ \\
\hline No (cat. ref.) & 728 & 22,0 & & & \\
\hline \multicolumn{6}{|c|}{ Participa en entidades orientadas al público en general } \\
\hline Sí & 428 & 12,9 & $-0,289$ & $-0,760 *$ & $-0,423$ ** \\
\hline No (cat. ref.) & 2883 & 87,1 & & & \\
\hline \multicolumn{6}{|l|}{ Documentación } \\
\hline Reside legalmente en España & 2960 & 89,4 & $-0,236$ & $-1,495^{* *}$ & $-0,626$ ** \\
\hline No reside legalmente en España (cat. ref.) & 351 & 10,6 & & & \\
\hline \multicolumn{6}{|l|}{ Periodo de llegada a España } \\
\hline 1990-96 & 378 & 11,4 & $-1,022$ ** & $-0,303$ & $-0,732$ ** \\
\hline 1997-01 & 1417 & 42,8 & $-0,566$ ** & $-0,425^{*}$ & $-0,146$ \\
\hline 2002-07 (cat. ref.) & 1516 & 45,8 & & & \\
\hline Constante & & & $-1,225^{* *}$ & $-0,235$ & 0,183 \\
\hline Casos totales & & & 3311 & 3311 & 3311 \\
\hline Varones empleados en cada nicho & & & 216 & 239 & 1229 \\
\hline $\mathbf{R}^{2}$ de Nagelkerke & 0,373 & & & & \\
\hline
\end{tabular}


Tabla 4.

Resultados de los análisis multinomiales para las mujeres inmigrantes

\begin{tabular}{|c|c|c|c|c|c|}
\hline & & & ostelería & $\begin{array}{l}\text { Peones } \\
\text { agrícolas }\end{array}$ & $\begin{array}{l}\text { Empleadas } \\
\text { del hogar }\end{array}$ \\
\hline Variables independientes & Casos & $\%$ & $\beta$ & $\beta$ & $\beta$ \\
\hline \multicolumn{6}{|l|}{ País de origen } \\
\hline Francia & 53 & 1,8 & $-1,452^{*}$ & & $-2,380 *$ \\
\hline Rumanía & 379 & 12,9 & 0,224 & 0,920 * & $0,513^{* *}$ \\
\hline Marruecos & 73 & 2,5 & 0,093 & $1,658^{\star *}$ & $-0,351$ \\
\hline Argentina & 156 & 5,3 & 0,016 & & $-0,773^{* *}$ \\
\hline Ecuador & 403 & 13,7 & $-0,227$ & $1,017^{\star *}$ & 0,089 \\
\hline Resto de inmigrantes (cat. ref.) & 1873 & 63,8 & & & \\
\hline \multicolumn{6}{|l|}{ Vive en pareja } \\
\hline Sí & 1739 & 59,2 & $-0,415^{\star *}$ & 0,474 & $-0,688$ ** \\
\hline No (cat. ref.) & 1198 & 40,8 & & & \\
\hline \multicolumn{6}{|l|}{ Ocupación en origen } \\
\hline $\begin{array}{l}\text { Hostelería } \\
\text { Hon }\end{array}$ & 234 & 8,0 & $0,925^{\star *}$ & 0,587 & 0,276 \\
\hline Agricultura & 47 & 1,6 & $0,844+$ & $1,972^{\star *}$ & $0,647+$ \\
\hline Empleada del hogar & 131 & 4,5 & 0,269 & 1,119 * & $0,888^{* *}$ \\
\hline Otra ocupación (cat. ref.) & 2525 & 86,0 & & & \\
\hline \multicolumn{6}{|l|}{ Es este su primer trabajo en España } \\
\hline Sí & 1020 & 34,7 & $-0,275^{*}$ & $-0,054$ & $0,517^{* *}$ \\
\hline $\begin{array}{l}\text { No (cat. ref.) } \\
\text { Nivel de estudios }\end{array}$ & 1917 & 65,3 & & & \\
\hline Sin estudios & 187 & 6,4 & 0,158 & $0,917+$ & 0,440 * \\
\hline $\begin{array}{l}\text { Primarios } \\
\text { Pans }\end{array}$ & 368 & 12,5 & 0,086 & $1,579^{* \star}$ & $0,421^{\text {** }}$ \\
\hline Secundarios $1 \mathrm{er}{ }^{0}$ & 394 & 13,4 & $0,320+$ & $1,314^{* *}$ & 0,215 \\
\hline Secundarios $2^{\circ}$ (cat. ref.) & 1229 & 41,8 & & & \\
\hline \multirow{2}{*}{\multicolumn{6}{|c|}{ Estudios en España o titulo convalidado }} \\
\hline & & & & & \\
\hline $\begin{array}{l}\text { Sí } \\
\text { No (cat ref) }\end{array}$ & $\begin{array}{r}352 \\
2585\end{array}$ & $\begin{array}{l}12,0 \\
88,0\end{array}$ & $-0,724^{* *}$ & $-1,464$ & $-0,870^{* *}$ \\
\hline \multicolumn{6}{|l|}{ Domina el español } \\
\hline Sí & 2654 & 90,4 & 0,066 & $-0,477$ & $0,463^{* *}$ \\
\hline No (cat. ref.) & 283 & 9,6 & & & \\
\hline \multicolumn{6}{|l|}{ Tenía contactos al llegar a España } \\
\hline Sí & 2473 & 84,2 & 0,157 & $-0,564+$ & $0,365^{* *}$ \\
\hline No (cat. ref.) & 464 & 15,8 & & & \\
\hline \multicolumn{6}{|c|}{ Participa en entidades orientadas al público en general } \\
\hline Sí & 382 & 13,0 & $-0,339+$ & $-1,185$ & $-0,337^{*}$ \\
\hline № (cat. ref.) & 2555 & 87,0 & & & \\
\hline \multicolumn{6}{|l|}{ Documentación } \\
\hline Reside legalmente en España & 2513 & 85,6 & $0,338+$ & 0,325 & $-0,421^{* *}$ \\
\hline \multirow{2}{*}{\multicolumn{6}{|c|}{ Periodo de llegada a España }} \\
\hline & & & & & \\
\hline $\begin{array}{l}1990-96 \\
1997-01\end{array}$ & 292 & 9,9 & $-0,342$ & a & $-0,606^{* *}$ \\
\hline $\begin{array}{l}\text { 1997-01 } \\
2002-07 \text { (cat. ref.) }\end{array}$ & 1170 & 39,8 & $-0,139$ & $-0,506+$ & $-0,381$ ** \\
\hline $\begin{array}{l}2002-07 \text { (cat. ref.) } \\
\text { Constante }\end{array}$ & 1475 & 50,2 & & & \\
\hline Constante & & & $-1,380^{* *}$ & $-3,624^{* *}$ & $-0,632^{\star \star}$ \\
\hline \multirow{3}{*}{$\begin{array}{l}\text { Casos totales } \\
\text { Mujeres empleadas en cada nicho } \\
R^{2} \text { de Nagelkerke }\end{array}$} & & \multirow{3}{*}{\multicolumn{2}{|c|}{$\begin{array}{r}2937 \\
413\end{array}$}} & 2937 & 2937 \\
\hline & & & & 66 & 807 \\
\hline & 0,228 & & & & \\
\hline
\end{tabular}

a No hay casos de individuos de esta categoría en este nicho laboral

${ }^{* * *} p<0,001{ }^{* *} p<0,01{ }^{*} p<0,05+p<0,1$ 
dio, sólo las rumanas presentan probabilidades significativamente superiores a las del resto de inmigrantes de emplearse en esta ocupación. Este nicho resulta especialmente atractivo para aquellas mujeres que buscan su primer empleo en España y que no tienen o no conviven con sus parejas. Como en el resto de los casos, tienden a concentrarse en este nicho étnico aquellas mujeres sin estudios o con estudios a nivel de primaria, así como quienes carecen de una convalidación de los estudios que han cursado en sus países de origen. En esta ocasión, y a diferencia de lo observado en el resto de ocupaciones, el dominio del castellano sí parece un factor que favorece la presencia de las mujeres inmigrantes en este nicho ocupacional. El acceso a contactos al llegar a España también aumenta significativamente la probabilidad de trabajar como empleada del hogar. No obstante, las mujeres que trabajan en este nicho muestran una propensión muy inferior a la del resto de mujeres inmigrantes a participar en asociaciones o entidades de atención al público. Los coeficientes de las variables que reflejan el estatus legal y el periodo de llegada a España sugieren que el trabajo doméstico concentra de manera muy particular a mujeres de llegada reciente y sin permiso de residencia.

Finalmente, cabe añadir que la ocupación en origen ha sido introducida en los modelos como una variable de control, pues a la hora de calcular los coeficientes de otras variables conviene tener en cuenta cuántos inmigrantes se emplean en un nicho étnico porque se han visto "forzados" a cambiar de ocupación al llegar a España o si, por el contrario, ya desempeñaban una ocupación similar en sus países de origen. En este sentido, comprobamos que esta variable juega también un papel importante. Entre las mujeres, el haber trabajado en el país de origen en el servicio doméstico propicia que en España también se encuentre empleo en esta misma ocupación o en el trabajo agrícola. Haber sido peón agrícola en origen aumenta las probabilidades de emplearse en España en cualquiera de los nichos étnicos identificados. Finalmente, el haber trabajado en los servicios hosteleros en origen aumenta de forma significativa la probabilidad de emplearse en España en una ocupación similar. Consideramos que este dato sustenta la idea de que el trabajo en hostelería goza de una mayor "deseabilidad" entre las mujeres inmigrantes que cualquiera de los otros dos. Entre los hombres sucede algo parecido: aquellos que han trabajado en servicios hosteleros tienden a concentrarse únicamente en la hostelería, mientras que los que cuentan con experiencia laboral en la construcción o la agricultura son significativamente más propensos a concentrarse en cualquiera de estas dos ocupaciones, pero no en la hostelería. Si relacionamos las variables "contactos", dominio del idioma nativo y la situación documental obtendremos un nuevo indicio de que, al menos entre las mujeres inmigrantes, no todos los nichos étnicos tienen el mismo grado de "deseabilidad". Los resultados de los modelos muestran que las mujeres que tienen contactos y dominan el idioma castellano tienden a trabajar como empleadas del hogar y a "huir" del trabajo en la agricultura. Por su parte, aquellas cuya situación documental está en regla y ya tienen una cierta experiencia laboral en España son más propensas a emplearse en los servicios de hostelería. 


\section{Conclusiones}

El primero de los dos objetivos de este artículo ha sido establecer las principales pautas de concentración étnica en el mercado laboral español según género y el país de origen. Hemos constatado que los varones superan a las mujeres en cuanto a la proporción de trabajadores insertados en nichos étnicos. Por otra parte, el análisis de los diferentes patrones de distribución en los nichos de varones y mujeres sugiere que la segregación por género se superpone a la segregación étnica. Seguidamente, los resultados han puesto en relieve que los varones y mujeres de origen francés se concentran principalmente en empleos de "cuello blanco". El nicho étnico en el que se ubican los argentinos es la hostelería y, en el caso de las mujeres, también el servicio doméstico. Entre los inmigrantes ecuatorianos, marroquíes y rumanos resaltan sus similitudes tanto en cuanto a los nichos ocupados como a las pautas de segregación por género. Los varones de estas tres procedencias han creado nichos de carácter étnico en la construcción. Por otra parte, los nichos femeninos son los servicios hosteleros, el servicio doméstico y la limpieza en oficinas/hoteles. En este sentido, los resultados de la ENI-2007 se muestran coherentes con las informaciones de otras fuentes estadísticas (como la Encuesta de Población Activa) y con los resultados de la investigación en España (Del Río y AlonsoVillar 2010). Más allá del análisis específico de los cinco colectivos de inmigrantes analizados, se puede apuntar que los datos confirman la existencia de modelos distintos de inserción laboral según el origen.

El segundo objetivo que nos hemos propuesto en este artículo ha sido determinar los factores de inserción en algunos de los nichos étnicos identificados previamente. En este sentido, hemos enunciado distintas hipótesis desde los planteamientos del capital humano, del capital social y de las teorías de la segmentación del mercado de trabajo. Los resultados confirman la validez de la hipótesis basada en el enfoque del capital humano. Tanto los varones como las mujeres con un nivel de preparación inferior (personas sin estudios y con estudios primarios) sufren un mayor riesgo de ocupar puestos de peones agrícolas, o de trabajar en la construcción o en el servicio doméstico. Teniendo en cuenta que el trabajo en estos tres nichos se basa, sobre todo, en el trabajo físico y en el desempeño de tareas que no requieren altos niveles de preparación ${ }^{12}$, podemos hablar de un fenómeno de ajuste entre la demanda del mercado laboral y la productividad medida en los niveles de formación. Por otra parte, el hecho de que el dominio del

\footnotetext{
${ }^{12}$ Entre los tres nichos mencionados, probablemente el trabajo en la construcción sea el que implica un desempeño (al menos en algunos puestos) de mayor complejidad de tareas. En este caso se debe tener en cuenta la peculiaridad del trabajo en este sector en el que las cualificaciones se obtienen mas a pie de obra que a través de la formación reglada. Como observa el Colectivo IOE (1998: 105) en referencia al trabajo en la construcción: "se constata que la cualificación laboral no depende de la formación profesional reglada,(...) la mayoría de los trabajadores que cumple tareas cualificadas no ha realizado ningún curso de formación. Esta desconexión entre formación reglada y cualificación de los trabajadores significa que las únicas oportunidades de aprendizaje se producen a través de la transmisión directa de conocimientos"
} 
español influya en que las mujeres inmigrantes se encuentren trabajando como empleadas del hogar también se puede interpretar como la consecuencia de un proceso de adaptación a una demanda específica de este nicho, pues esta ocupación requiere un mejor conocimiento del idioma, dado el continuo contacto con los miembros del hogar (Martínez 2004).

Las hipótesis del capital social quedan tan sólo parcialmente confirmadas. El tener contactos en España en el momento de la llegada sí parece favorecer la inserción de los hombres en la construcción, y entre las mujeres, propicia su entrada en el servicio doméstico. Sin embargo, nuestra suposición de que el acceso a los contactos más heterogéneos disminuye el riesgo de encontrarse en un nicho étnico sólo se ha confirmado entre las mujeres en la hostelería y en el servicio doméstico y entre los varones en la agricultura y en la construcción.

También hemos hallado pruebas de la importancia de los factores contextuales que relacionamos con los procesos de segmentación del mercado laboral. El haber llegado a España en un periodo de mayor crecimiento económico favorece la inserción laboral de los varones en todos los nichos estudiados, si bien en el caso de las mujeres, tan sólo propicia su entrada en el servicio doméstico. Por otra parte, carecer de un permiso de residencia aumenta la probabilidad de que un inmigrante se emplee en la construcción o como peón agrícola entre la población masculina, o como empleada del hogar entre la población femenina. Cabe subrayar que los tres sectores mencionados se caracterizan por una alta presencia de la economía sumergida, lo que viene a reafirmar la relación entre las regulaciones administrativas y los fenómenos de segregación en el mercado laboral.

Nuestro estudio indica adicionalmente que no todos los nichos étnicos tienen el mismo grado de "deseabilidad", sino que existe una jerarquía en la que el trabajo agrícola aparece como el menos atractivo. Suponemos que los inmigrantes van construyendo trayectorias (sociales) a través de itinerarios laborales, mejorando su situación a través de esos nichos jerarquizados y, posteriormente, fuera de ellos. Cabe subrayar, no obstante, que los datos sólo dejan apuntar esta realidad dado el relativamente corto periodo de tiempo que la mayoría de las poblaciones analizadas llevan en España.

También se pone de relieve que la inserción en nichos étnicos responde a distintos mecanismos de asignación dependientes del género. Nuestros análisis indican que el trabajo agrícola es un nicho en el que se concentran aquellos varones con menos recursos tanto en términos de capital humano como en cuanto a su situación documental. Entre las mujeres, en cambio, encontrarse en situación regular y poseer estudios al nivel de secundaria de primer grado no protege ante el riesgo de incorporarse en este nicho. Finalmente, el hecho de que la inserción en dos nichos exclusivos de cada sexo (construcción para los varones y trabajo doméstico para las mujeres) esté vinculada a casi el mismo conjunto de predictores (con algunas diferencias concretas) confirma que en el mercado laboral español operan otros determinantes de segregación que influyen en las pautas de división del trabajo por género (Balcells 2009; Ibáñez 2008). 


\section{REFERENCIAS BIBLIOGRÁFICAS}

Abad, L. V. 2002. "Trabajadores inmigrantes en las economias avanzadas. La paradoja de la demanda adicional en mercados con exceso de oferta." Pp. 459-467 en II Congreso sobre la inmigración en España. La inmigración en España. Contextos y alternativas, compilado por J. García Castaño y C. Muriel López. Granada: Universidad de Granada.

Alcobendas, M. A. y N. Rodríguez-planas. 2009. “Immigrants' assimilation process in a segmented labor market." IZA Discussion Paper Series IZA Dissusion Paper Number: 4394. Institute for the Study of Labor.

Arjona, A. y J. C. Checa. 2006. "Economía étnica. Teorías conceptos y nuevos avances." Revista Interna-cional de Sociología 64: 117-143.

Balcells, L. 2009. "Analyzing the division of household labor within Spanish families." Revista Internacional de Sociología 67: 83-105.

Bernardi, F., L. Garrido y M. Miyar. 2011. "The recent fast upsurge of immigrants in Spain and their employment patterns and occupational attainment." International Migration 49: 148-187.

Bradley, H. y G. Healy. 2008. Ethnicity and gender at work: inequalities, careers and employment relations. Houndmills: Palgrave Macmillan.

Cachón, L. 2009. La 'España inmigrante': marco discriminatorio, mercado de trabajo y políticas de integración. Barcelona: Anthropos Editorial.

Canal-Domínguez, J. F. y C. Rodríguez-Gutiérrez. 2008. "Analysis of wage differences between native and immigrant workers in Spain." Spanish Economical Review 10: 109-134.

Castles, S. y M. J. Miller. 2003. The Age of Migration: International Population Movements in the Modern World (3rd ed.). Nueva York: Palgrave Macmillan.

Cebolla, H. y A. González. 2008. La inmigración en España (2000-2007). De la gestión a la integración de los inmigrantes. Madrid: Centro de Estudios Politicos y Constitucionales.

Colectivo IOE. 1998. Inmigración y trabajo: trabajadores inmigrantes en el sector de la construcción: (polacos y marroquíes en Madrid y Barcelona). Madrid: Instituto de Migraciones y Servicios Sociales.

Cuadrado, J. R., C. Iglesias y R. Llorente. 2007. Inmigración y mercado de trabajo en España (19972005). Madrid: Fundación BBVA.

Chiswick, B. R., L. Lee y P. W. Miller. 2003. "Patterns of Immigrant Occupational Attainment in a Longitudinal Survey." International Migration 41: 47-69.

Del Río, C. y O. Alonso-Villar. 2010. "Occupational segregation of immigrant women in Spain." 2010165. ECINEQ Working Papers. Society for the Study of Economic Inequality.

Domingo, A., F. Gil-Alonso y G. Robertson. 2007. "Immigration and changing labour force structure in the southern European Union." Population (English Edition) 62: 709-727. 
Ellis, M. y R. Wright. 1999. "The industrial division of labor among immigrants and internal migrants to the los angeles economy." International Migration Review 33: 26-54.

Fernández, C. y C. Ortega. 2008. "Labor market assimilation of immigrants in Spain: employment at the expense of bad job-matches?" Spanish Economical Review 10: : 83-107.

Friedberg, R. M. 2000. "You can't take it with you? Immigrant assimilation and the portability of human capital." Journal of Labor Economics 18: 221-251.

Garrido, L. y L. Toharia. 2004. "La situación laboral de los españoles y los extanjeros según la Encuesta de Población Activa." Economistas 22: 74-86.

Green, G. P., L. M. Tigges y D. Diaz. 1999. "Racial and Ethnic Differences in Job-Search Strategies in Atlanta, Boston, and Los Angeles." Social Science Quarterly 80: 263-278.

Hudson, M. 2003. "Modeling the probability of niche empoyment: exploring workforce segmentation in metropolitan Atlanta." Urban Geography 23: 528-559.

Ibáñez, M. 2008. "La segregación ocupacional por sexo a examen. Características personales, de los puestos y de las empresas asociadas a las ocupaciones masculinas y femeninas." Revista Española de Investigaciones Sociológicas 123: 87-122.

Light, I. 2007. "Economías étnicas." Pp. 41-68 en Empresariado étnico en España, compilado por B. Joaquín, L. Oso y N. Ribas. Madrid: CIDOB; MTAS.

Martín Urriza, C. 2006. "Una comparación entre el salario de los trabajadores españoles y extranjeros." Revista de Ministerio de Trabajo y Asuntos Sociales 61: 61-75.

Martínez, U. 2004. Trabajadores invisibles. Precariedad, rotación y pobreza de la inmigración en España. Madrid: Catarata.

Martori, J. C. y K. Hoberg. 2004. "Indicadores cuantitativos de segregación residencial. El caso de la población inmigrante en Barcelona." Scripta Nova. Revista Electrónica de Geografía y Ciencias Sociales VIII. (169), http://www.ub.edu/geocrit/sn/sn-169.htm.

Massey, D. S. 2002. "Residential Segregation." Pp. 348-354 en A companion to racial and ethnic studies, compilado por D. T. Goldberg y J. Solomos. Maklen: Wiley-Blackwell.

Massey, D. S., R. Alarcon, J. Durand y H. González. 1987. Return to aztlan: the social process of international migration from western México. Berkeley: University of California Press.

McGovern, P. 2007. "Immigration, labour markets and employment relations: problems and prospects." British Journal of Industrial Relations 45: 217-235.

Merschrod, K. 1981. "The index of dissimilarity as a measure of inequality." Quality and Quantity 15: 401-411.

Model, S. 1993. "The Ethnic Niche and the Structure of Opportunity: Immigrants and Minorities in New York City." Pp. 161-193 en The Underclass Debate: Views from History, compilado por M. Katz. Princeton: Princeton University Press. 
Model, S. 1997. "Ethnic Economy and Industry in Mid-Twentieth Century Gotham." Social Problems 44: 445- 463.

Morawska, E. 1990. "The sociology and historiography of immigration." Pp. 187-238 en Immigration Reconsidered: History, Sociology, and Politics, compilado por V. Yans-McLaughlin. Nueva York: Oxford University Press.

Ooka, E. y B. Wellman. 2003. "Does Social Capital Pay Off More Within or Between Ethnic Groups? Analysing Job Searchers in Five Toronto Ethnic Groups." Pp. 199-226 en Inside the Mosaic, compilado por E. Fong. Toronto: Univeristy of Toronto Press.

Pajares, M. 2010. Inmigración y mercado de trabajo. Informe 2010. Madrid: Ministerio de Trabajo e Inmigración.

Parella, S. 2003. Mujer, inmigrante y trabajadora: la triple discriminación. Barcelona: Anthropos.

Pascual, À., V. de Miguel y M. Solana. 2007. Redes sociales de apoyo. La inserción de la población extranjera. Bilbao: Fundación BBVA.

Pedace, R. 2006. "Immigration, labor market mobility, and the earnings of native-born workers. An occupational segmentation mpproach." American Journal of Economics and Sociology 65: 313-345.

Piore, M. 1979. Birds of passage: migrant labour and industrial societies. Cambridge: Cambridge University Press.

Portes, A. 1998. "Social capital: Its origins and applications in modern sociology." Annual Review of Sociology 24: 1-24.

Raijman, R. y M. Semyonov. 1995. "Modes of labor market incorporation and occupational cost among new immigrants to Israel." International Migration Review 29: 375-394.

Rath, J. 2002. "A quintessential immigrant niche? The non-case of immigrants in the Dutch construction industry." Entrepreneurship and Regional Development 14: 355-377.

Rosenfeld, M. J. y M. Tienda. 1999. "Mexican immigration, occupational niches, and Labor-Market Competition: Evidence from Los Angeles, Chicago and Atlanta, 1970-1990." Pp. 64-105 en Immigration and Opportunity: Race, Ethnicity, and Employment in the United States, compilado por F. D. Bean y S. Bell-Rose. Nueva York: Russell Sage Foundation.

Sanders, J. M., V. Nee y S. Sernau. 2002. "Asian immigrants' reliance on social ties in the multiethnic labor market." Social Forces 81: 281-314.

Schrover, M., J. Van Der Leun y C. Quispel. 2007. "Niches, Labour Market Segregation, Ethnicity and Gender." Journal of Ethnic and Migration Studies 33: 529-540.

Simón, H., E. Sanromá y R. Ramos. 2008. "Labour segregation and immigrant and native-born wage distributions in Spain: an analysis using matched employer-employee data." Spanish Economical Review 10: 135-168.

Stanek, M. y A. Veira. 2009. "Occupational transitions and social mobility at migration to Spain." Documentos de Trabajo GEPS 3(4). Grupo de Estudios Población y Sociedad. Madrid. 
Valdez, Z. 2006. "Beyond the ethnic enclave: The effect of ethnic solidarity and market opportunity on white, Korean, Mexican and Black Enterprise." Pp. 341-385 en Crossing Borders/Constructing Boundaries: Race, Ethnicity, and Immigration, compilado por C. Brettell. Landham: Lexington Books.

Vidal, E., F. Gil y A. Domingo. 2009. "The Non-EU-25 female population in Spain: A factor analysis of labour market integration at regional level." Pp. en Labour markets and demographic change, compilado por M. Kuhn y C. Ochsen. Wiesbaden: VS Verlag für Sozialwissenschaften.

Waldinger, R. 1996. Still the promised city? African Americans and new immigrants in postindustrial Nueva York. Cambridge: Harvard University Press.

Wang, Q. 2004. "Labour market concentration of Asian ethnic groups in US metropolitan areas: a disaggregated Study." Population, space and place 10: 479-494.

Wang, Q. y K. Pandit. 2007. "Measuring Ethnic Labour Market Concentration and Segmentation." Journal of Ethnic and Migration Studies 33: 1127-1252.

Wilson, F. D. 1999. "Ethnic concentrations and labor-market opportunities." Pp. 106-140 en Immigration and opportunity: Race, ethnicity, and employment in the United States, compilado por F. D. Bean y S. Bell-Rose. Nueva York: Russell Sage Foundation.

Wilson, F. D. 2003. "Ethnic niching and metropolitan labor markets." Social Science Research 32: 429-466.

Wright, R. y M. Ellis. 2000. "The ethnic and gender division of labor compared among immigrants to Los Angeles." International journal of urban and regional research 24: 583-600.

ALBERTO VEIRA RAMOS es Profesor ayudante doctor en el Departamento de Sociología y Ciencias Políticas de la Universidad Carlos III de Madrid. Obtuvo el título de Master of Sciences en sociología por la Universidad de Wisconsin-Madison (EEUU) gracias a una beca de la Fundación Caixa Galicia y posteriormente se doctoró en sociología por la Universidad Nacional de Educación a Distancia. Ha sido durante nueve meses investigador predoctoral en el Institut National d'Études Démographiques (INED) de París gracias a una beca de la Xunta de Galicia y de la Fundación Caixa Galicia, e investigador posdoctoral en el Institut für Soziologie de la Georg-August Universität de Göttingen durante 18 meses gracias a una beca para la movilidad del MICINN. Sus principales áreas de investigación son la demografía del mercado laboral en una perspectiva comparada y los métodos de análisis cuantitativos.

MIKOLAJ STANEK es Investigador postdoctoral en el Consejo Superior de Investigaciones Científicas y miembro del Grupo de Estudios Población y Sociedad. Su área de mayor interés son los procesos de inserción laboral de los inmigrantes y las migraciones desde Europa Central y Oriental. Actualmente forma parte del proyecto Encuesta Nacional de Inmigrantes (ENI): Explotación con Perspectivas Comparativas Nacionales e Internacionales. Entre sus publicaciones más recientes se encuentran: Flujos migratorios desde Europa Central y Oriental después de 1989 en Historia y Politica 2010, Inmigrantes ucranianos en España - una aproximación a las pautas de movilidad internacional en Scripta Nova 2010 (con R. Hosnedlová) y Patterns of Romanian and Bulgarian migration to Spain, Europe Asia Studies 2009.

LORENZO CACHÓN es Catedrático de Sociología de la Universidad Complutense de Madrid y miembro del GEMI (Grupo de Estudios sobre Migraciones Internacionales). Sus líneas de investigación son: Inmigración y mercado de trabajo; Políticas de inmigración y de integración de los inmigrantes; 
Discriminación y políticas antidiscriminatorias y Conflictos ligados a la inmigración. Entre sus última publicaciones se encuentran: (director) Inmigración y conflictos en Europa: aprender para una mejor convivencia (Hacer, 2011); (autor) La "España inmigrante": marco discriminatorio, mercado de trabajo y políticas de integración (Anthropos, 2009); (editor con M. Laparra), Inmigración y políticas sociales (Bellaterra, 2009); (autor) Bases sociales de los sucesos de Elche de septiembre de 2004. Crisis industrial, inmigración y xenofobia (2005).

\section{RECIBIDO: $17 / 11 / 2010$}

ACEPTADO: 11/01/2011 